\title{
A novel video-assisted anatomic segmentectomy technique: Selective segmental inflation via bronchofiberoptic jet followed by cautery cutting
}

Morihito Okada, MD, PhD, ${ }^{\text {a }}$ Takeshi Mimura, MD, ${ }^{\text {a }}$ Junichi Ikegaki, MD, PhD, ${ }^{\text {b }}$ Hiromi Katoh, MD, PhD, Harumi Itoh, MD, PhD, ${ }^{\mathrm{c}}$ and Noriaki Tsubota, MD, $\mathrm{PhD}^{\mathrm{a}}$

From the Departments of Thoracic Surgery $^{\mathrm{a}}$ and Anesthesiology, ${ }^{\mathrm{b}}$ Hyogo Medical Center for Adults, Akashi City, Hyogo, and the Department of Radiology, ${ }^{\mathrm{c}}$ Fukui Medical University, Yoshida, Fukui, Japan.

Informed consent was obtained from all the patients.

Received for publication Aug 26, 2006; revisions received Oct 22, 2006; accepted for publication Nov 2, 2006.

Address for reprints: Morihito Okada, $\mathrm{MD}, \mathrm{PhD}$, Department of Thoracic Surgery, Hyogo Medical Center for Adults, Kitaohjicho13-70, Akashi City 673-8558, Hyogo, Japan (E-mail: morihito1217jp@aol.com).

J Thorac Cardiovasc Surg 2007;133:753-8

$0022-5223 / \$ 32.00$

Copyright (C) 2007 by The American Association for Thoracic Surgery

doi:10.1016/j.jtcvs.2006.11.005
Objective: Segmentectomy is an anatomic parenchyma-sparing resection that is recently being performed for small-sized lung carcinoma and constitutes a useful procedure in a thoracic surgeon's armamentarium. We have generated a new technique that improves the identification of the intersegmental border and whose clinical utility we evaluate in this study.

Methods: Under bronchofiberscopy, jet ventilation is selectively applied to the burdened bronchus to develop an anatomic plane between the inflated segment to be resected and the deflated area to be preserved. From April 2004 to June 2006, 52 consecutive patients with a clinical T1 N0 M0 peripheral cancer $2 \mathrm{~cm}$ or smaller underwent video-assisted segmental resection called hybrid VATS segmentectomy in which electrocautery with no stapler was used to divide the intersegmental plane detected by selective jet ventilation.

Results: Complete resection was achieved in all patients. The median operative time and bleeding during the operation were 155 minutes (range 85-225 minutes) and 60 $\mathrm{mL}$ (range 10-210 $\mathrm{mL}$ ), respectively. The complication rate was $13.5 \%$ (7/52), and the most common was concerning air leak. The median duration of postoperative air leak and chest tube drainage was 1 day and 3 days, respectively. There were no in-hospital deaths. There was one case of mediastinal lymph node recurrence and another of metastasis to the brain although there was no case of local recurrence in the surgical margin area.

Conclusions: A novel video-assisted segmentectomy technique for lung cancer is clinically useful. Selective segmental inflation provides an obvious intersegmental plane quickly and easily, allowing a real margin distance in the ventilated segment. Despite the minimally invasive approach, since only the segment to be resected and not the entire lobe is expanded, an appropriate surgical view is possible.

$\mathrm{P}$ ulmonary segmentectomy was first carried out for bronchiectasis in the lingula by Churchill and Belsey in 1939 and had been performed since then in many patients with an infectious disease such as tuberculosis and in selected patients with non-small cell lung carcinoma (NSCLC). However, in the past few decades the frequency of this procedure has considerably decreased because of the advent of antibiotic agents for infections, the improvement of sanitary conditions, the outcome of the randomized study by the Lung Cancer Study Group, ${ }^{2}$ demonstrating that sublobar resections including wedge resections resulted in higher rate of local recurrence than did lobectomy in patients with stage IA NSCLC, and the escalating incidence of nonanatomic stapled wedge resection. Consequently, most recent residency programs of thoracic surgery do not include segmentectomy as an obligatory procedure, but several expert thoracic surgeons have advanced that 


\section{Abbreviations and Acronyms \\ NSCLC $=$ non-small cell lung cancer \\ VATS $=$ video-assisted thoracic surgery}

segmentectomy is important as a basic surgical technique and should not be forgotten by current-generation thoracic surgeons. ${ }^{3-7}$

In concordance with the explosive increase of early detected small-sized NSCLCs through the development of radiographic tools and the widespread practice of screening, ${ }^{8}$ we have been aggressively performing radical segmentectomy with lymph node assessment not only in high-risk patients but also in good-risk patients with clinical stage IA tumors $2 \mathrm{~cm}$ or smaller. ${ }^{9-12}$ Lately, we have used a new method to detect the intersegmental plane in segmentectomy that involves selective jet ventilation under bronchofiberscopy. With this method the segment to be removed can be inflated while keeping the segments to be preserved without air. This technique is completely opposite to the conventional method and allows the clear visualization of the intersegmental line between the segment to be resected and the one to be preserved. We can see the real surgical margin in the inflated segment and can get a good surgical field even through video-assisted thoracic surgery (VATS) without having to make an effort to suppress the other segments and lobes with an instrument. In addition, dissection of the anatomic intersegmental plane by electrocautery but not by staple reduces local failure at the margin, one of the unfavorable recurrent patterns, and makes the preserved adjacent segments fully expansive to obtain maximum pulmonary function. In this study, we describe this novel technique through hybrid VATS approach ${ }^{13}$ in detail and evaluate its clinical utility.

\section{Materials and Methods}

\section{Surgical Procedure of Segmentectomy}

Patients are positioned in the lateral decubitus position and the lung is isolated with a double-lumen endotracheal tube. Basically, two skin incisions are built for access with no extracostal muscles or ribs cut. One utility incision for manipulation and another access port for the insertion of a thoracoscope are placed. The surgeon directly observes the hilum of the diseased lobe and individually isolates and severs all the bronchi and vessels, although television monitor guidance is variably used during the procedure when dissecting an area out of direct view, which is called hybrid VATS. ${ }^{13}$ More recently, the main access has been opened with a wound retractor made of silicone rubber with no rib spreading. It is crucial to note that the skin incision must be extended without hesitation if the surgeon has difficulty with the surgical view. We favor a backhand grip, holding 30-cm-long scissors (model 1018098-30; Mayo-Harrington; Stille, Sweden) for sharp dissection, lengthy needle holders, and forceps upside-down. Pleural lavage cytology is examined immediately after the introduction of a thoracoscope to check the subclinical tumor dissemination. ${ }^{14}$

Recognition of the intersegmental plane is accomplished by differential inflation with jet ventilation. After the segmental bronchi are isolated, an anesthesiologist puts a $3.5-\mathrm{mm}$ bronchofiberscope through the double-lumen tube into the orifice of the targeted segmental bronchus. The tip of the bronchofiberscope is recognized at the surgical field because the surgeon can see the light of the tip and lead it to the suitable place of the targeted bronchus, in which high-frequency oscillation is started $(40 \mathrm{~Hz}$, working pressure $2 \mathrm{~kg} / \mathrm{cm}^{2}$, HFO Jet Ventilator; Mera, Tokyo, Japan). The diseased segment is inflated while the preserved segments appear collapsed, and a line is formed between the inflated and the deflated lung parenchyma, evidencing the anatomic intersegmental plane. ${ }^{11}$ This technique allows the development of a defined plane without air transmission through collateral ventilation, unlike the conventional method. After jet ventilation fills the targeted segment, the distal site of the bronchus is tied to keep the segment inflated, and the proximal site to the tie is transected, leaving a stump of sufficient length so that closure will not occlude other segmental orifices. When more than one segment is to be removed, the surgeon can selectively insert the tip of the fiberscope into each segmental bronchus and inflate one segment after the other. At the central portion around the hilum, the intersegmental plane is approached along the intersegmental vein, and at the peripheral site electrocauterization is used along the inflation-deflation line. With a commercially available fibrin sealant (Bolheal; Chemo-Sero Therapeutic Institute, Kumamoto, Japan), composed of fibrinogen and thrombin and an absorbable polyglycolic acid felt (Neoveil; Japan Medical Planning Co, Kyoto, Japan), the raw surface of the remaining lung prevents air leakage after cutting by cautery. Only when the lung is emphysematous can the surgeon use staplers for dividing the intersegmental plane to keep air leak to a minimum. Since a margin greater than the tumor diameter, that is, at least $2 \mathrm{~cm}$ of healthy lung tissue, is required, the resection line can be placed on the segment adjacent to the affected one, or portions of a few adjacent segments or subsegments can be extirpated (Figure 1). Sampling or dissection of segmental, lobar, hilar, and mediastinal lymph nodes followed by frozen-section analysis is mandatory to decide the applicability of segmentectomy. In patients with intentional indication for radical segmentectomy, lobectomy should be performed instead when the surgical margin is judged to be imperfect or any lymph node is found to be diseased. Routinely, the chest is drained with a single chest tube under water seal, which is inserted through the incision initially established for the thoracoscope.

\section{Patients}

Between April 2004 and June 2006, 52 consecutive patients with a clinical T1 N0 M0 peripheral NSCLC tumor of $2 \mathrm{~cm}$ or less in every dimension on high-resolution computed tomography underwent segmentectomy. Electrocautery with no stapler, not even partially, was used to divide the intersegmental plane. Resected specimens, especially the surgical margin, were examined histopathologically, and histologic typing was done according to the World Health Organization classification. ${ }^{15}$ Surgical-pathologic staging was performed according to the New International Staging 
$\mathbf{A}$

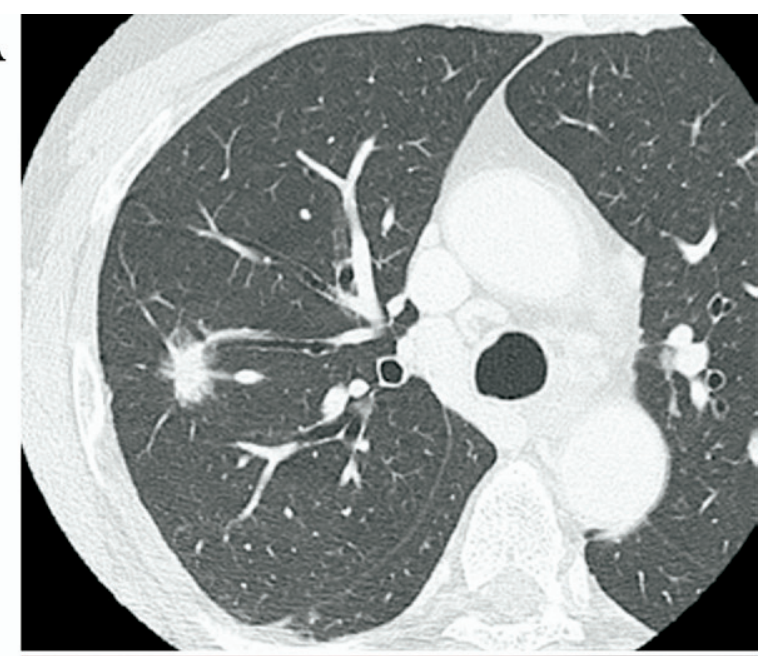

B

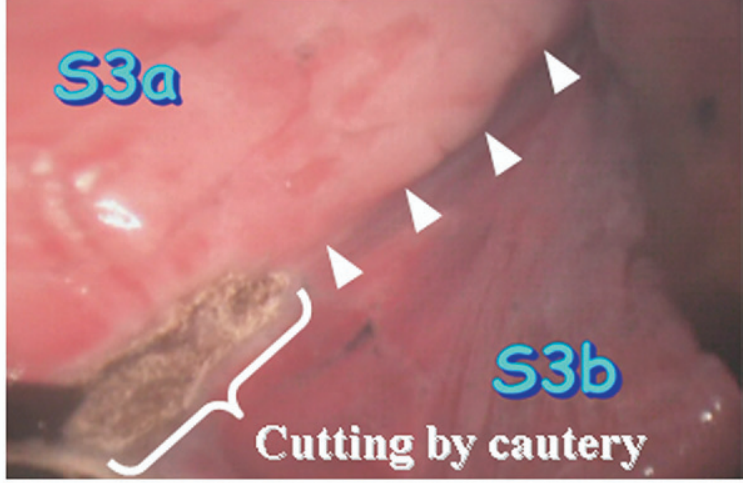

C

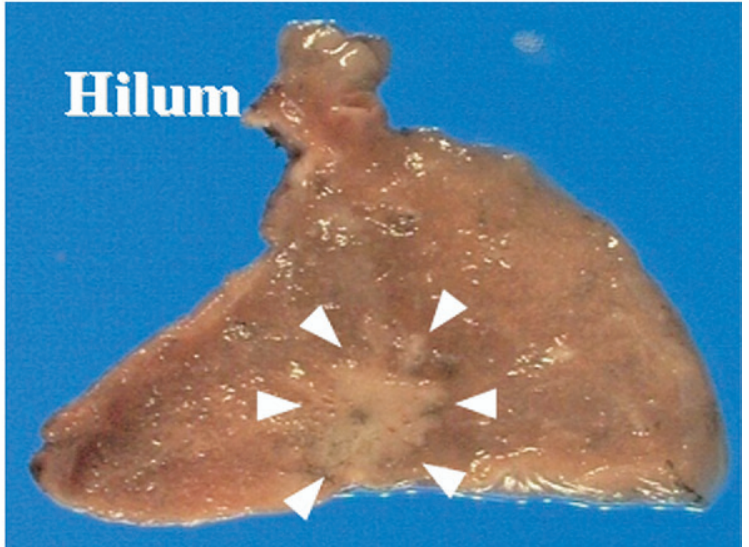

Figure 1. S2b (anterior subsegment of posterior segment) + S3a (posterior subsegment of anterior segment) resection of the right upper lobe, a representative of atypical segmentectomy. A, Highresolution computed tomographic image showing a tumor located at the border between S2b and S3a. B, Intraoperative findings showing the inflation-deflation line (arrowheads) between the inflated (resected) S3a and the deflated (preserved) S3b segments. C, Resected surgical specimen showing the tumor (arrowheads) with sufficient margin.

\section{TABLE 1. Patient characteristics}

\begin{tabular}{lc}
\hline No of patients & 52 \\
Age (y) & $40-82$ \\
Range & 65 \\
Median & \\
Sex & 27 \\
$\quad$ Male & 25 \\
Female & \\
Size of tumor (mm) & $8-20$ \\
Range & 18 \\
Median & \\
Histology & 45 \\
Adenocarcinoma & 29 \\
$\quad$ Including bronchioloalveolar carcinoma component & 5 \\
Squamous cell & 1 \\
Large cell & 1 \\
Carcinoid & \\
Pathologic & 49 \\
Stage IA & 2 \\
Stage IB & 1 \\
Stage IIA & \\
\hline
\end{tabular}

System for Lung Cancer. ${ }^{16}$ Each patient provided his or her informed written consent based on the protocol approved by the institute's review board before the operation. After the operation, every patient was basically evaluated at 3-month intervals for the first 2 years and at 6-month intervals thereafter.

\section{Results}

The characteristics of the patients are listed in Table 1. Among the 52 patients, there were 25 women and 27 men with a median age of 65 (range 40-82) years. Tumor size ranged from 8 to $20 \mathrm{~mm}$ (median size $18 \mathrm{~mm}$ ). Histologically, the vast majority of the patients had an adenocarcinoma $(45 / 52,86.5 \%)$, a high proportion of which contained a bronchioloalveolar carcinoma component (29/45, 64.4\%). Pathologic examination showed that curative resections were achieved with free surgical margins in all patients.

Table 2 shows the locations of burdened segments. Upper lobe lesions predominated, with right upper lobe tumors being the most common. Segmentectomy can actually be performed in any lobe, excluding the middle lobe. The operative results are shown in Table 3 . The median operative time measured from skin incision to skin closure and median bleeding during the operation were 155 minutes (range 85-225 minutes) and $60 \mathrm{~mL}$ (range 10-210 $\mathrm{mL}$ ), respectively. The median skin incision for the utility access was $65 \mathrm{~mm}$ (range 40-120 mm). Postoperative complications occurred in 7 patients $(13.5 \%)$, the most common one being prolonged air leak. One patient had a late alveolopleural fistula 5 days after the chest tube had been removed on the third postoperative day and required tube drainage for 4 days. Another patient had a minor alveolopleural fistula on the sixth day, which healed without requiring tube 
TABLE 2. Location of burdened lung

$\begin{array}{lr}\begin{array}{l}\text { No. of patients } \\ \text { Lobe }\end{array} & 52 \\ \text { Upper } & 29 \\ \text { Lower } & 23 \\ \text { Resection } & \\ \text { Right } & 27 \\ \text { S1 } & 3 \\ \text { S1 }+2 a & 1 \\ \text { S2 } & 5 \\ \text { S2b }+3 a & 4 \\ \text { S3 } & 3 \\ \text { S6 } & 8 \\ \text { S6 }+8 & 1 \\ \text { S7+8 } & 1 \\ \text { S }+10 & 1 \\ \text { Left } & 25 \\ \text { S1 }+2 a & 1 \\ \text { S1 }+2+3 & 7 \\ \text { S } 4+5 & 5 \\ \text { S } 6 & 7 \\ \text { S } 8 & 2 \\ \text { S } 9+10 & 3\end{array}$

$a$, posterior subsegment; $b$, anterior subsegment; $S 1$, apical; $S 2$, posterior; $S 3$, anterior; $S 4$, superior; $S 5$, inferior; $S 6$, superior; $S 7$, medial basal; $S 8$, anterior basal; $S 9$, lateral basal; $S 10$, posterior basal.

drainage. In $22(42.3 \%)$ patients no air leak was observed at the time of surgery. The duration of postoperative air leak and chest tube drainage ranged from 0 to 11 days (median, 1 day) and 2 to 13 days (median, 3 days), respectively. There was no case of in-hospital death or 30-day mortality. During the follow-up period (median 16 months; range 2-28 months), we experienced one case of local recurrence in the mediastinal lymph node and another of distant metastasis to the brain, but there was no case of local recurrence in the surgical margin.

\section{Discussion}

The development of radiographic devices such as highresolution computed tomography and the widespread practice of low-dose helical computed tomography for screening $^{8}$ have resulted in an amazing increase of early detection of ever-smaller NSCLCs that possibly have a more indolent biologic behavior, such as bronchioloalveolar carcinoma. The trend has rapidly been changing clinical practice in thoracic surgery and, accordingly, many surgeons have without doubt become concerned over the unified treatment with whole lobectomy for these small peripheral cancers. Removing a relatively large volume of healthy lung tissue may result in a poorer quality of postoperative life, a higher frequency of operative morbidity, and fewer possibilities to additionally have a second or even a third NSCLC resected, for which these patients would survive long
TABLE 3. Surgical results

\begin{tabular}{lc}
\hline No. of patients & 52 \\
Operation time (min) & $85-225$ \\
Range & 155 \\
Median & \\
Bleeding (mL) & $10-210$ \\
Range & 60 \\
Median & \\
Utility access incision (mm) & $40-120$ \\
$\quad$ Range & 65 \\
$\quad$ Median & \\
Complication & 4 \\
$\quad$ Prolonged air leak (>7 d) & 2 \\
Late alveolopleural fistula* & 1 \\
Supraventricular arrhythmia & \\
Air leak duration (d) & $0-11$ \\
Range & 1 \\
Median & \\
Chest tube duration (d) & $2-13$ \\
Median & 3 \\
Range &
\end{tabular}

*0ccurring after removal of the chest tube.

enough to be at risk. Recently, several authors have reported that sublobar resection was not inferior to lobectomy concerning the prognosis of selected patients with a small-sized NSCLC. ${ }^{9-12,17-20}$

The Lung Cancer Study Group study showed a higher occurrence of local recurrence after sublobar resection, ${ }^{2}$ and we noted that their series included a high proportion of wedge resections in the sublobar resection group (32.8\%, 40/122) for tumors up to $3 \mathrm{~cm}$ in diameter; thus we think that the predominance of wedge resection might have influenced the frequency of local recurrence. In contrast, the percentage of wedge resections was $11.5 \%$ (30/260) in our previous report, ${ }^{12}$ which targeted tumors up to $2 \mathrm{~cm}$. If the indication had been limited to tumors of $2 \mathrm{~cm}$ or smaller and if segmentectomy, which could improve the treated margin, had commonly been used, the frequency of local recurrence could have been lower..$^{9-12,17,18}$ Additionally, we are afraid that the more frequent application of wedge resection might lead to a lower assessment of lymph nodes, leading to a potential understaging of the disease, contrary to segmentectomy, which allows the inspection of the status of regional lymph nodes. We believe that segmentectomy is anatomic resection and should be completely distinguished from nonanatomic wedge resection. Nowadays, segmental resections are hardly being performed and the procedure has become unfamiliar to many thoracic surgeons. Particularly when intentionally planning a radical sublobar resection, the surgeon must overcome the great temptation to perform an easier wedge resection. The development of stapling devices has made huge wedge resection without regard to anatomic planes an almost overwhelming alternative to segmen- 
tectomy, despite warnings that the distortion of the stapled residual lobe might lead to pleural complications such as empyema and fistula and loss of lung function. Segmentectomy also allows an optimal resection of suspicious smallsized deep-seated lesions with safe surgical margins, since adopting diagnostic lobectomy for a potential underlying malignancy must be avoided.

In general, segmentectomy is technically trickier than lobectomy, requiring deep 3-dimensional knowledge of the relevant bronchoarterial relationships and possible anomalies of arterial branches. Sharp dissection by scissors accurately and quickly exposes the segmental hilar structures. Habitually, the arterial branches are initially divided, allowing identification of the segmental bronchus, which is the most reliable landmark of a segment because of its rare anomaly. The ligation of segmental vein is best performed last, after the intersegmental plane has been outlined, as the venous drainage might not be actually apparent. Identification of the intersegmental plane, which basically exists in lobes (Figure 2), is performed by differential inflation, in which the diseased segment is selectively inflated by jet ventilation and thus demarcated, quickly producing an inflation-deflation line. It is a great advantage to accurately define the real margin distance provided in an inflated diseased segment and to limit expansion to only the selected segment, not to the entire lobe, for obtaining an appropriate surgical field in this era of VATS. Generally, the reverse procedure is used; occlusion of the segmental bronchus in an airless whole lung is followed by expansion of the lung. Collateral ventilation through Kohn's pores will often fill the diseased segment owing to the positive air pressure used in this method, which accordingly fails to outline the inflationdeflation line. The main cause of the failure is relatively strong positive pressure, which is required to inflate the whole lung. In contrast, the pressure of jet ventilation is absolutely weak to selectively inflate only the targeted segment. During jet ventilation the surgeon can see the gradual inflation of the targeted segment, adjust the pressure of the jet ventilation under his or her direct vision, and stop the jet ventilation at the time of complete inflation before air flows into the collateral ventilation. Intriguingly, the intersegmental plane is approached along the inflation-deflation line at the peripheral site while the intersegmental vein is a landmark at the central portion around the hilum. Although sparing the intersegmental veins that delineate the perimeter of a bronchopulmonary segment and drain contiguous segments, and consequently preserving the venous drainage of adjacent segments, is a requirement of segmental resection to keep the full function of the adjacent segments, the surgeon should not hesitate to remove the intersegmental vein when the margin from the tumor is considered insufficient.

Dissection of the segmental plane by electrocautery is strongly recommended because it offers some advantages
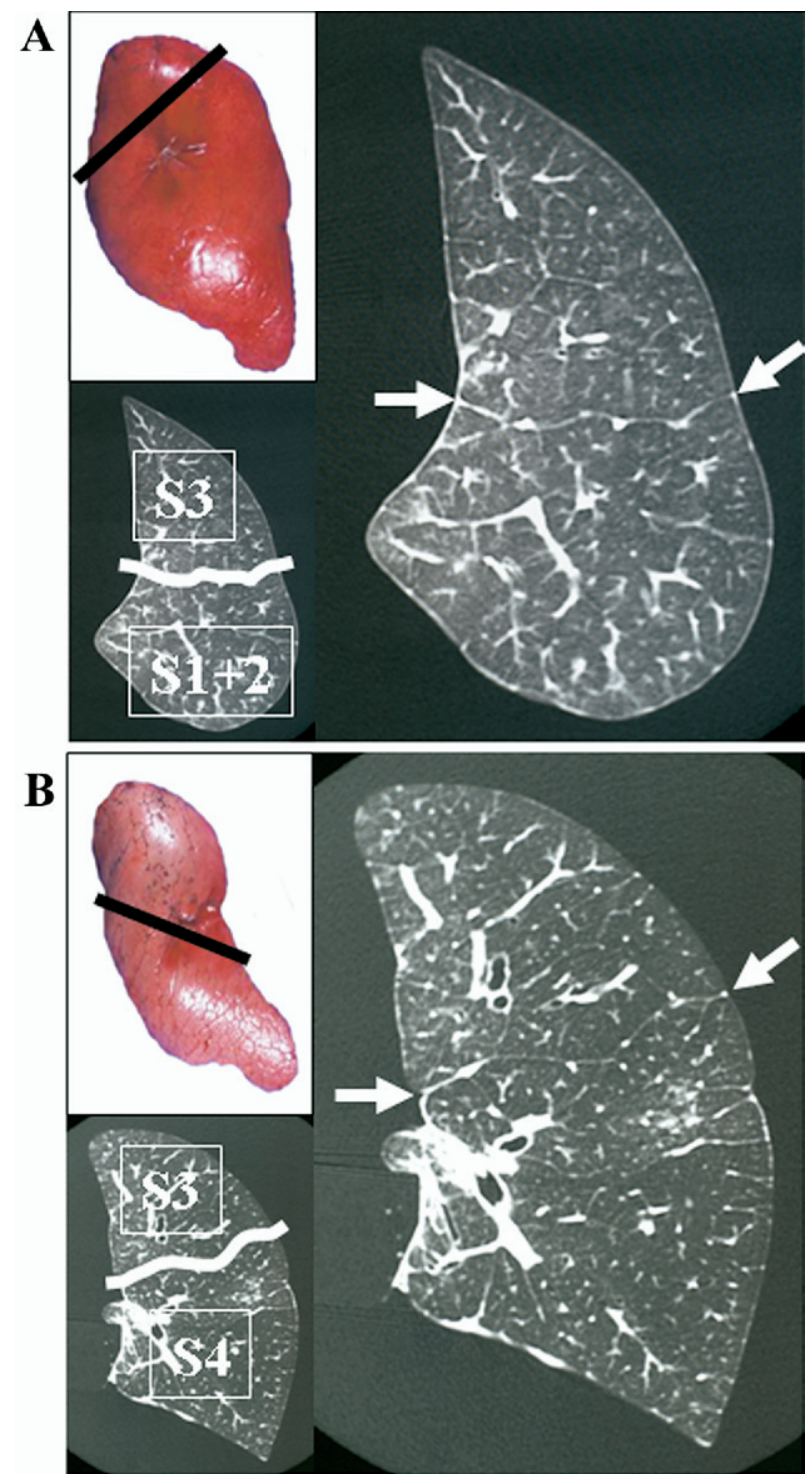

Figure 2. Transaxial (A) and coronal (B) views of specimen computed tomographic image in the left upper lobe, showing the anatomic intersegmental lines between $S 1+2$ (apical and posterior segments) and $S 3$ (anterior segment) (A), as well as between S3 and S4 (superior segment) (B), respectively. These findings are considered evidence of definitely outlined inflation-deflation lines.

over that by staplers: not only can the surgeon extirpate deep-seated tumors or tumors existing in locations where a stapler cannot be applied, but he or she also can freely cut the lung parenchyma to have a sufficient margin, which does not necessarily require afterward checking for residual tumor cells on the cutting planes. If any staple is used, the surgical margin could possibly be more reduced than expected and thus should be investigated. In addition, the 
application of stapling devices can often compromise adjacent pulmonary parenchyma, restricting full expansion of the residual segments and thus pulmonary function, a major goal of segmental resection. However, unerring use of staples may be advantageous in patients with emphysema for stringent control of air leak. In the present study, air leak was a delicate issue after segmental resection. Small alveolopleural fistulas may seal, leaving a neutral air space that usually reabsorbs with gradual expansion of the lung because the residual space after segmentectomy is much smaller than that after lobectomy.

\section{References}

1. Churchill ED, Belsey R. Segmental pneumonectomy in bronchiectasis. Ann Surg. 1939;109:481-99.

2. Lung Cancer Study Group, Ginsberg RJ, Rubenstein LV. Randomized trial of lobectomy versus limited resection for T1N0 non-small cell lung cancer. Ann Thorac Surg. 1995;60:615-22.

3. Jensik RJ. Miniresection of small peripheral carcinomas of the lung. Surg Clin North Am. 1987;67:951-8.

4. Breyer RH, Zippe C, Pharr WF, Jensik RJ, Kittle CF, Faber LP. Thoracotomy in patients over age seventy years: ten-year experience. J Thorac Cardiovasc Surg. 1981;81:187-93.

5. Warren WH, Faber LP. Segmentectomy versus lobectomy in patients with stage I pulmonary carcinoma: five-year survival and patterns of intrathoracic recurrence. J Thorac Cardiovasc Surg. 1994;107:1087-93.

6. Stair JM, Womble J, Schaefer RF, Read RC. Segmental pulmonary resection for cancer. Am J Surg. 1985;150:659-64.

7. Jones DR, Stiles BM, Denlinger CE, Antippa P, Daniel TM. Pulmonary segmentectomy: results and complications. Ann Thorac Surg. 2003;76:343-9.

8. Patz EF Jr, Goodman PC, Bepler G. Screening for lung cancer. $N$ Engl J Med. 2000;343:1627-33.
9. Okada M, Yoshikawa K, Hatta T, Tsubota N. Is segmentectomy with lymph node assessment an alternative to lobectomy for non-small cell lung cancer of $2 \mathrm{~cm}$ or smaller? Ann Thorac Surg. 2001;71:956-61.

10. Yoshikawa K, Tsubota N, Kodama K, Ayabe H, Taki T, Mori T. Prospective study of extended segmentectomy for small lung tumors: the final report. Ann Thorac Surg. 2002;73:1055-8.

11. Tsubota N, Ayabe K, Doi O, Mori T, Namikawa S, Taki T, et al. Ongoing prospective study of segmentectomy for small lung tumors. Study Group of Extended Segmentectomy for Small Lung Tumor. Ann Thorac Surg. 1998;66:1787-90.

12. Okada M, Koike T, Higashiyama M, Yamato Y, Kodama K, Tsubota N. Radical sublobar resection for small-sized non-small cell lung cancer: a multicenter study. J Thorac Cardiovasc Surg. 2006; 132:769-75.

13. Okada M, Sakamoto T, Yuki T, Mimura T, Miyoshi K, Tsubota N. Hybrid surgical approach of video-assisted minithoracotomy for lung cancer: significance of direct visualization on quality of surgery. Chest. 2005;128:2696-701.

14. Okada M, Sakamoto T, Nishio W, Uchino K, Tsuboshima K, Tsubota N. Pleural lavage cytology in non-small cell lung cancer: lessons from 1000 consecutive resections. J Thorac Cardiovasc Surg. 2003;126: 1911-5.

15. World Heath Organization histologic typing of lung tumours. 2nd ed. Am J Clin Pathol. 1982;77:123-36.

16. Mountain CF. Revisions in the International System for Staging Lung Cancer. Chest. 1997;111:1710-7.

17. Kodama K, Doi O, Higashiyama M, Yokouchi H. Intentional limited resection for selected patients with T1 N0 M0 non-small cell lung cancer. J Thorac Cardiovasc Surg. 1997;114:347-53.

18. Koike T, Yamato Y, Yoshiya K, Shimoyama T, Suzuki R. Intentional limited pulmonary resection for peripheral T1 N0 M0 small-sized lung cancer. J Thorac Cardiovasc Surg. 2003;125:924-8.

19. Martin-Ucar AE, Nakas A, Pilling JE, West KJ, Waller DA. A casematched study of anatomical segmentectomy versus lobectomy for stage I lung cancer in high-risk patients. Eur J Cardiothorac Surg. 2005;27:675-9.

20. Keenan RJ, Landreneau RJ, Maley RH Jr, Singh D, Macherey R, Bartley S, et al. Segmental resection spares pulmonary function in patients with stage I lung cancer. Ann Thorac Surg. 2004;78:228-33. 